\title{
Study on Improving Resource Sharing in Higher Education Park by Grid Technology
}

\author{
He Yongqiang* \\ College of Computers \\ Henan Institute of Engineering \\ Zhengzhou, China \\ e-mail: yqhe18@126.com \\ * Corresponding Author
}

\author{
Qin Qin \\ College of Computers \\ Henan Institute of Engineering \\ Zhengzhou, China \\ e-mail: qq@haue.edu.cn
}

\begin{abstract}
It has already been the key problem for development of current higher education that how to allocate educational resource in the higher education park to realize resource sharing and how to make full use of the educational resource in the park to improve scientific and sustainable development of it. It is necessary to put forward new models and techniques to avoid various problems met before and to solve the problem of educational resource sharing fundamentally. This paper mainly analyses how to realize integration and interoperation of heterogeneous resources by means of grid technology from the perspective of technology. Moreover, it emphasizes on discussion of methods and prospects of applying grid technology to educational field for the purpose of realizing sharing and construction of resources in Higher Education Parks.
\end{abstract}

Keywords- grid technology; Higher Education Park; resource sharing; architecture; management model

\section{INTRODUCTION}

Rapid development of social economy of China requires that education should be popularized. In addition, there is great demand for higher education from the society and individuals. It has been an important way to meet this requirement by enlarging educational scale. Thereupon, building new campuses is a realistic option. A new phenomenon of higher education, namely the Higher Education Park, has appeared in many cities in China driven by several factors including guidance of the government and educational requirements of colleges and universities.

The essential purpose of building the Higher Education Park is to realize maximum utilization rate of higher educational resources through resource sharing, improve educational efficiency and accelerate improvement of educational quality of universities while extending scale of higher education. It is obvious that resource sharing is the goal to be achieved by the Higher Education Park. At present, upgrading and renewing of hardware environment of network and emerging of various new techniques provide new methods for solving the problem of educational resource sharing, among which, grid technology has advantages like being in different management domains and being in favor of realizing mutual accessibility of dynamic heterogeneous resource in wide range, which can be helpful to build a new resource sharing and construction model.

\section{ARCHITECTURE OF GRID}

Application of grid can be divided into data-intensive and compute-intensive type, which can also be called data grid and compute grid respectively. As one of the important methods of resource sharing, data gird emphasizes on storage, distribution and duplicate policy of data. Moreover, bottom-level data can be heterogeneous, in different management domains and even dynamic, which are of great advantage to realize integration and sharing of resources without changing too much of the existing data running platform.

Determination of grid architecture is the premise to realize application of it. Every aspect involved in grid technology has gradually formed its own regulations and protocols. Building grid system in strict accordance with these normalized techniques is the key to realize general-purpose nature and interoperability of data. According to ideas of Ian Foster, grid can be shown in Five-Level Sandglass Architecture, as shown in Fig. 1.

A. Fabric

Fabric is the bottom level, which acts as hardware basis for grid building, including all kinds of computing resources (i.e. supercomputer and precious instruments), storage resources, network resource, code library, directory and so on. Meanwhile, in view of the above mentioned resources, the fabric level support program starts, monitor and process controls in terms of computing resources; in terms of storage resource, it supports storage and access of files, high-performance transfer, selection and reduction of remote data as well as control and management mechanism of resources allocated for data transfer; in terms of transmission resources of network, it can provide management mechanism and query function to get information of network characteristic and load. In addition, the fabric level realizes the management mechanism of source code and object code, supports query and updating of directory or relation database, as well as reserves resources in advance. In conclusion, by means of the aforementioned mechanisms, the fabric level realizes controlling, management and scheduling of local resources in local range. 


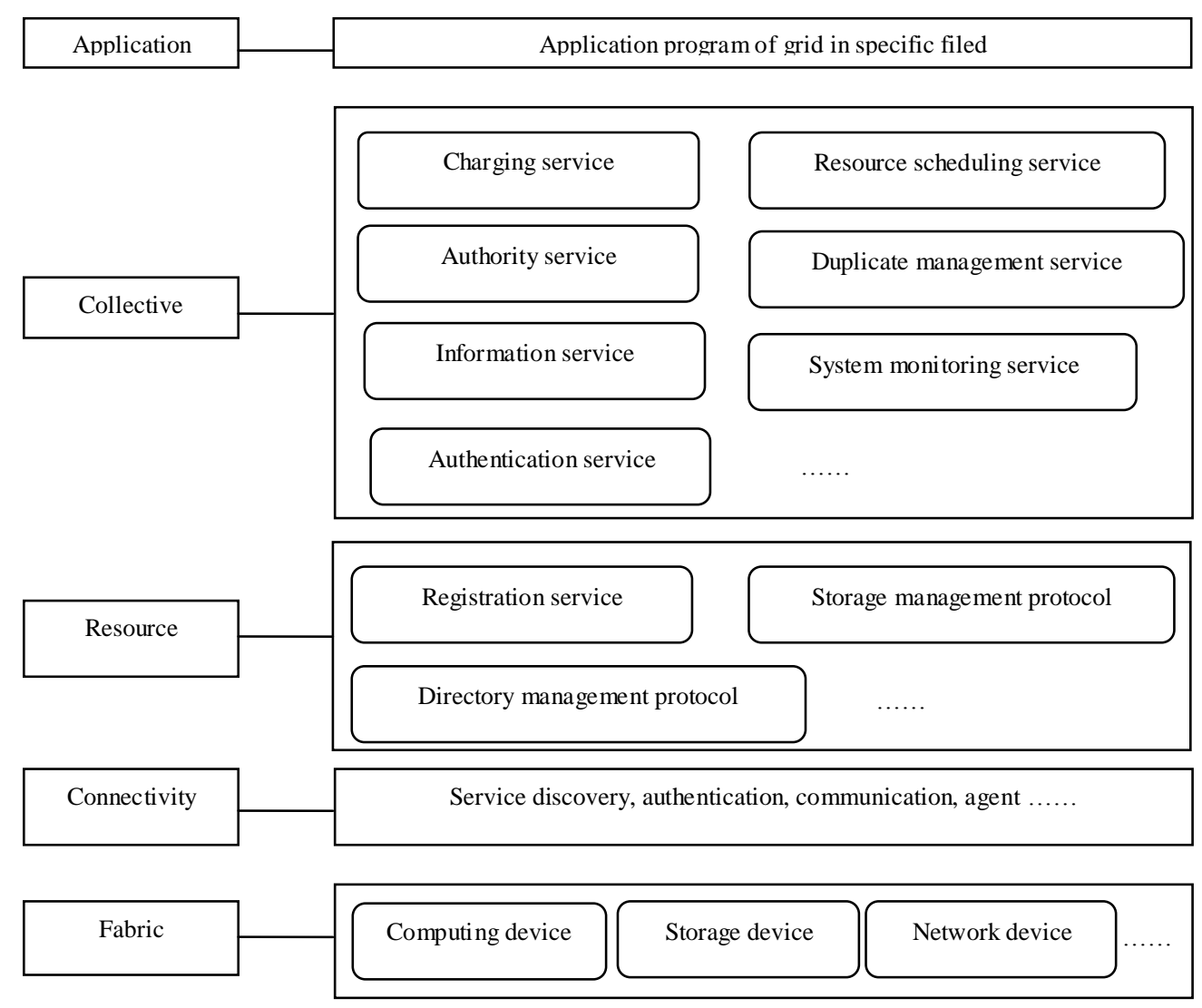

Figure 1. Hierarchical chart of grid

\section{B. Connectivity}

Connectivity is above the fabric level. This level defines the communication protocol and validation protocol (the existing internet protocol also included) involved in running of grid for the purpose of realizing data communication among different platforms. One of the important problems to be solved by the connectivity level is the security certification of communication. Only by ensuring validity and safety of data in the bottom level can the application in the upper level realize in right manner. Usually, the one-time verification method for safety certification is adopted, in other words, the users have to pass the system verification after logging in for one time, and then they have access to different grid resources or local mapping or integration by using this legal identity. Security mechanism has been set within local range of each heterogeneous grid resource, so interoperation between the security mechanism of the whole grid and local mechanism must be achieved and integration must be realized by means of mapping. Furthermore, access to resources in different domains can be realized by building trust mechanism among users.

\section{Resource}

Resource mainly offers various normalized protocols and interfaces for management and operation of resources, among which there are some application program interfaces (API) and software package. Topological features and status information (such as system configuration, load, running model, etc) of various resources can be gained from the resource level. Moreover, resource level can monitor related operational process and achieve the functions like charging and payment as required.

\section{Collective}

Collective is to collect all kinds of resources. Specifically speaking, the collective level is responsible for reservation, cooperation and scheduling tasks produced when several resources are working. Besides, it also builds a series of services to support access to resources by upper-level application. The services are detailed as follows: directory service, that is to say build catalog information for resource for the benefit of application program in discovering and retrieval of resources; data reproduction service, which refers to that store data transcription in the system by use of certain duplicate policy to ensure speed and efficiency of access to data; resources scheduling service refers to allocate resources reasonably according to certain optimization objective to increase degree of satisfaction of users and system response; monitoring service is to monitor states of resources, including validity and load of resources.

\section{E. Application}

Application refers to the application program level for users in grid. Use application program to call the corresponding service through Application Programming Interface (API) of each level, thus to realize all kinds of functions, which can be unified into an integrated environment for development of grid and provide all kinds of tool software. 


\section{REALIZE EDUCATIONAL RESOURCE SHARING BY USE OF GRID}

Combining characteristics of educational resources, management model of educational resources can be built based on standard architecture of grid to integrate educational resources of each college and university into a simple and uniform resource sharing environment by use of grid technology. According to metadata code of educational resources, the bottom level data will be encapsulated as learning object and corresponding services will be designed to realize storage, query and registration of learning objects. Because WebService has been widely accepted, it is unnecessary to take heterogeneity of bottom level platform and data into consideration when the application program of upper level is established on service. In this way, seamless integration of heterogeneous data can be realized.

Building the uniform resource sharing platform shall be of advantage to integration of existing resources, what is more important is to provide normalized operating standard and corresponding tool software for creating new educational resources. In this way, quality and collecting efficiency of resources can be improved gradually, and augmentability and sustainability of the platform can be strengthened. Based on the purposes aforementioned, the educational resources sharing frame is put forward based on grid technology, which contains the following flow, such as the establishment at earlier stage, issue, organization and the management of educational resources. Functional levels are divided according to architecture of grid and interlayer interface is defined, as shown in Fig 2.

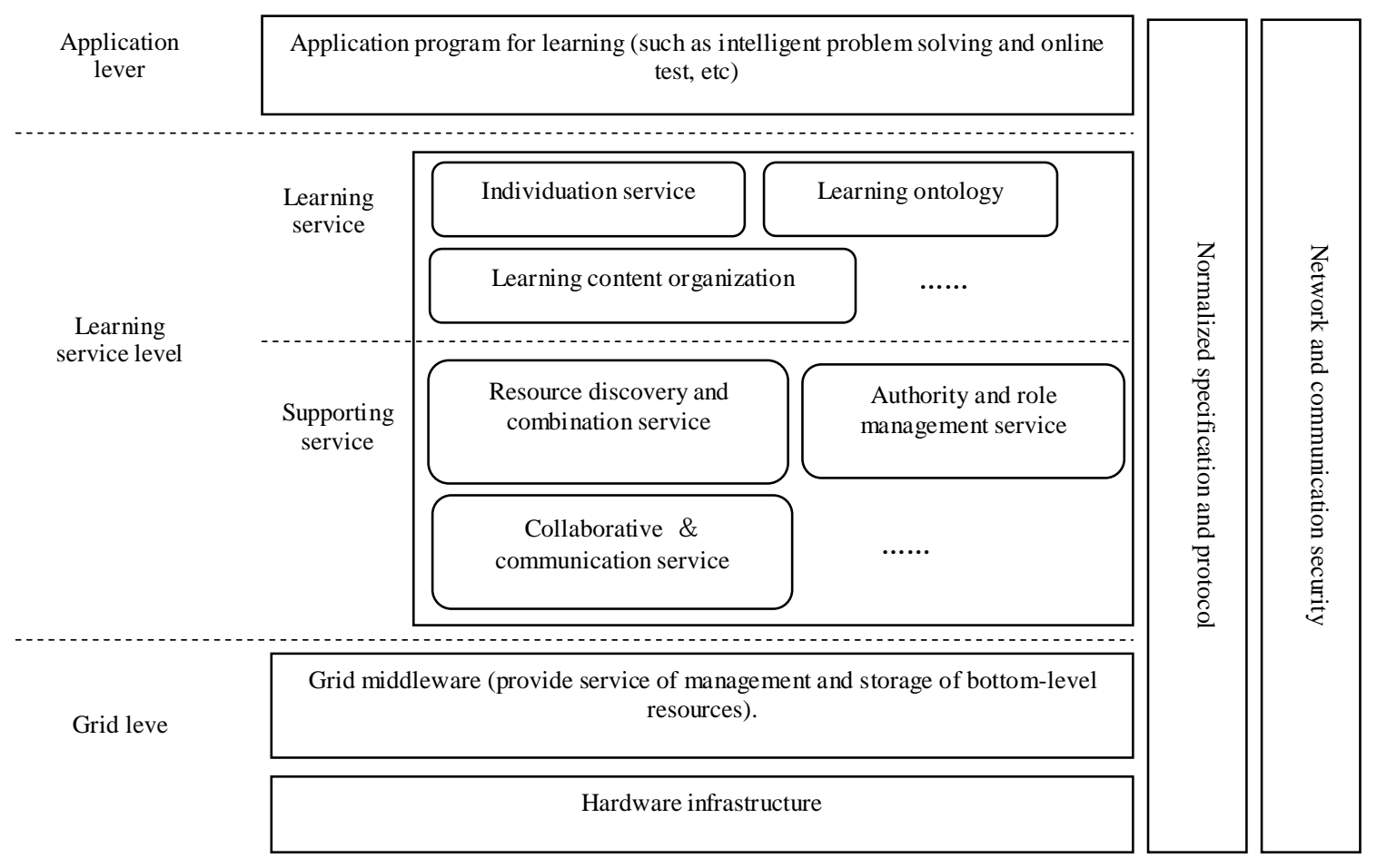

Figure 2. Frame diagram

\section{A. Resource Description Layer in Grid}

Educational resources include various teaching materials like texts, audio, video, animation and other large-grained resources like item bank, common question library and network courseware as well. In order to achieve resources sharing, as to the existing resources, the standardized Web Service can be used to mask the data format at bottom layer and platform differences, which will provide upper layer application with unified interface; as to the newly joined resources, XML and RDF are recommended to be adopted to describe metadata according to standard. If semantic application needs to be supported, OWL can be adopted to define the ontology.

Deployment of educational resources should be done in accordance with the statistic of system access. As to the educational resources with higher access frequency, it is necessary to ensure there is enough bandwidth or adopt certain data duplication technique to disperse access flow.
In addition, in order to facilitate the discovery and retrieval of resources, resources at all levels should provide the service of resources directory, and the access to directory can be achieved in the way similar to routing, that is to say, the positioning of resources can jump from certain level of directory to the next level until the resources is found in the end. It is noteworthy that resources description here does not only refer to the metadata description of the resources itself, but also refers to the description of resources state and service interface information provided by the resources. At the same time, the information of resources state also needs to be registered, so as to make it convenient for users to search and use. Since resources in the grid change dynamically, those registered information is also in a state of continuous updating.

\section{B. Learning Service Layer}

The main characteristics of storage and sharing of educational resources by grid, is that it has realized various 
services supporting educational application based on the grid middleware. These services mainly include: resources registration service, resources discovery service, resources directory service, resources retrieval service, resources access service, statistic analysis service, resources management service and standardized service. The above-mentioned services are used for the upper-level application, that is to say, the service components may be called by application developers. The realization of each service is built on the much lower layer of supporting service, such as duplicate management service, resources scheduling service, transmission control service and so on. Generally, the core service mainly achieves the following four functions: data access, storage, security authentication and information service. Data access service is mainly used for the unified interface which realizes the access to the distributed resources server. It can make users feel no difference when accessing to heterogeneous data, so as to be favorable to the sharing of educational resources. Storage service is mainly used for the management of transmission and storage of distributed data. At the same time, it needs to create data copy according to factors like system throughput and bandwidth changes. It also places these copies on the server according to certain strategy to improve the response speed of system. Security authentication service provides security guarantee for the legitimate use of educational resources in grid and authenticate the relevant operation if needed, for example, the identity authentication when login. In addition, it also supports the authority control when accessing data, charging function and digital certificate function. Information service mainly provides information like states and property of resources and relevant metadata for various application services. These information acts as the basis of some key service algorithms in grid, such as resources scheduling algorithm, dynamic balancing algorithm and service discovery algorithm, etc.

In addition, due to the dynamic changes of grid resources environment, new resources may join and overtime resources may quit on occasion. Thus, system must be able to perceive the existence of other resources, so as to provide users with better retrieve service. This task is mainly completed by resources discovery service. Based on the research on distributed resources storage model and combining the registration information of resources, resources discovery service has built the corresponding resources discovery mechanism, which makes resources access break the restriction of management domain and heterogeneous platform and provides unified interface for searching resources.

\section{Application}

Program developers can build various teaching application programs at the application level by applying services provided by learning service layer. These programs cannot only be the specific software for learning and management, but also be a teaching support platform which integrates all kinds of functions. As for the ordinary students, teachers or teaching managers, they don't need to know how various services are realized in the process of using grid system, but only need to learn the basic resource retrieve methods, then they can use the whole system resources just like using local resources.
Only to develop some traditional teaching application (such as tutoring and answering questions, online test and discussion group, etc.) is not enough, it is also necessary to provide a series of new teaching methods by making full use of the characteristics and advantages of grid technology. These characteristics and advantages include:

\section{1) Intelligent Retrieval Based on Semantic}

With the increase of resources amount, traditional keyword matching method becomes more and more difficult to find resources that are really necessary for users. However, in the process of integrating a variety of educational resources by using grid, semantic description information can be provided for the existing resources and build ontology, so as to make resources retrieval become more intellectualized and improve the satisfaction degree of users.

\section{2) Information Push Based on Scene}

In addition to the active search of resources by users, according to the tracking information of users' learning process, the system can push learning content to users in combination with the learning situation (such as time and place and so on). According to the modern educational concept, learning process can not be carried out through totally centering on students, it does need certain guidance. Therefore, in educational technology category, this teaching concept can be better realized by learning content push service.

\section{3) Large-Scale Collaborative Learning}

The cross-domain of grid and wide geographical distribution make the scope of cooperation and collaboration among learners expanded. It has been found that this kind of communication and discussion among users can make them better grasp the learning knowledge point and improve the learning effect.

Besides, various management functions must be provided by the application level, in order to make system complete all kinds of configurations and management functions without changing programs. Management includes role and authority management of system users, resources information management, auditing management and charging management.

In general, architecture of educational resources grid should include protocol specifications and middleware service required for realizing resources sharing. The transparency and security in the utilization process of resources should be taken into consideration from the perspective of users; the storage and management of resources and the support to management tools should be considered from the perspective of system management; charging authentication should be emphasized from the perspective of resources supplier, which can make the educational resources grid platform which is built in the end meet the actual needs, and promote the sharing and building of educational resources.

\section{CONCLUSIONS}

Currently, the Internet is in a period of evolution and transition to the next generation, which is also the best time for solving the sharing problem of educational resources. In the process of upgrading and updating their campus networks by colleges and universities and educational institutions, the technical proposal and route compatible with each other which are formed by them in 
advance, would be significant to the elimination of "information island" , the promotion of educational cooperation and the improvement of resources utilization ratio and education quality. Therefore, this paper puts forward the educational resources sharing mechanism and architecture taking grid as the basic platform. Making full use of the characteristics of grid technology which supports the heterogeneous resources integration and frees from geographical and regional restriction can be helpful to promote educational resources sharing in Higher Education Parks.

This research was supported by Science and Technology Project of Henan Science and Technology Office(NO.152102210027) and project of the Education Department of Henan Province (No. 15A520054). We would like to thank the referee for his/her careful reading and helpful suggestions.

\section{REFERENCES}

[1] XU Wen-qiang, GU Yun-hua: Research on grid-based CSCL resource sharing platform of university. Information Technology, 2010, (12).

[2] ZHAO Ying, YANG Hai-ying, ZHANG Zhi-jun: The research of resource mamagement model in grid service. Journal of Liaoning Normal Nniversity(Natural Science Edition), 2010, 33(4).

[3] LI Xiao-qing: Resource Management of Digital Library Based on Grid Environment. Library Tribune, 2009, 29(1).
[4] LI Jian-bo, CHEN Xiang: Research of model of resource management based on campus grid. Computer Engineering and Design, 2007, 28(19).

[5] He Yongqiang and Wang Xuerui, Study on Educational Resources Management System Based on Grid Technology, AISC 2012, V146.

[6] Gui Xiaolin, Grid Computing Technology, Beijing University of Posts and Telecommunications Press, 2005.

[7] YE Bing-liang, DAI Wenzhan, YANG Weiqi: Study on the Current Situation and Countermeasures of Laboratory Resources Sharing in Higher-Education Zone. Research and Exploration in Laboratory, 2006, 25(11).

[8] Zhang Xichun: Application of Data Mining in Information Resources Construction and Sharing within the Higher Education Zone. Journal of Library Science, 2010,32(12).

[9] Ding Donglan, Resource Sharing and Open Teaching Model,Zhejiang University Press, 2012.

[10] Maozhen Li, Mark Baker, The Grid core Technologies, Tsinghua University Press, 2006

[11] He Yongqiang and Zhang Shunli, Study on Co-allocation Algorithm of Educational Grid Resource, AISC 2012, V146.

[12] Zhang Zhiying, Theory and methods of professional evaluation of higher education, China Social Sciences Press, 2011.

[13] Zhuang Wenxian, Yang Xue, Zhou Yumin, Sun Jie: The Design of Regional Educational Resources Sharing System Based on Grid. Journal of Intelligence, 2010, 29(6). 